\title{
Anchoring high-throughput screening methods to scale-up bioproduction of siderophores
}

\author{
Marco P.C. Marques $^{\mathrm{a}, \mathrm{b}, *}$, Kieran Walshe ${ }^{\mathrm{c}}$, Sean Doyle ${ }^{\mathrm{c}}$, Pedro Fernandes ${ }^{\mathrm{a}, \mathrm{b}}$, Carla C.C.R. de Carvalho ${ }^{\mathrm{a}, \mathrm{b}}$ \\ a IBB-Institute for Biotechnology and Bioengineering, Centre for Biological and Chemical Engineering, Instituto Superior Técnico, Av. Rovisco Pais, 1049-001 Lisboa, Portugal \\ ${ }^{\mathrm{b}}$ Department of Bioengineering, Instituto Superior Técnico, Universidade Técnica de Lisboa, Lisboa, Portugal \\ c National Institute for Cellular Biotechnology, Department of Biology, National University of Ireland Maynooth, Maynooth, Co. Kildare, Ireland
}

\section{A R T I C L E I N F O}

\section{Article history:}

Received 22 April 2011

Received in revised form

24 November 2011

Accepted 25 November 2011

Available online 3 December 2011

\section{Keywords:}

Siderophore

Marine bacteria

High throughput

Process intensification platform

Image analysis

\begin{abstract}
A B S T R A C T
The use of high throughput strategies is of acknowledged relevance since the rational use of small-scale reactors, coupled with suitable analytic tools, is contributing to the acceleration of process development in several areas of biotechnology. These small-scale reactors are available in different working volumes and configurations, being useful in a wide array of applications, from cell screening to process optimization.

The present work was focused on the development of a high-throughput strategy, combining microtiter plates and analytic methodologies, to screen an in-house library of environmental bacteria in order to identify good siderophore producers. From a library of roughly 500 marine microorganisms, it was possible to ultimately obtain 11 bacterial strains with high production capabilities. Two of them had not been previously identified as siderophore producers. The bioprocess was scaled-up from microtiter plates to a $5 \mathrm{~L}$ stirred tank reactor, while maintaining the overall volumetric productivity, using the $k_{L} a$ similarity as scale-up criterion.

This novel approach is a suitable alternative to traditional screening tools.
\end{abstract}

(C) 2011 Elsevier Ltd. All rights reserved.

\section{Introduction}

Bacteria synthesize a vast diversity of chemical compounds, with applications ranging from the pharmaceutical to the food industries, e.g. therapeutic steroids [1], biofuels [2] and terpenoids [3]. The combination of the natural diversity with further synthetic modifications provides an unlimited resource for complex compounds unmatched by chemical synthesis $[4,5]$.

These biochemical and/or semi-biochemical routes are desirable in industry since they occur under mild conditions, reduce waste and require in general less toxic compounds, when compared to similar chemical routes. However, the potentially interesting compounds are usually produced by microorganisms in low quantities. Nonetheless, under suitable growth and/or reaction conditions the yield can be increased in most cases [6].

Traditional bioprocess development encompasses the identification of the envisaged biological activity on a given microorganism, and occasionally the use of a suitable expression system. The screening of the desired compound, as well as the optimization of operational and environmental conditions for its production,

\footnotetext{
* Corresponding author at: IBB-Institute for Biotechnology and Bioengineering, Centre for Biological and Chemical Engineering, Instituto Superior Técnico, Av. Rovisco Pais, 1049-001 Lisboa, Portugal. Tel.: +351 218419065.

E-mail address: mpc.marques@ist.utl.pt (M.P.C. Marques).
}

typically demands an intensive amount of experimental work. This can be performed either in agar plates or in shaken flasks and more recently in microtiter plates (MTP). The availability of process intensification platforms is therefore a great asset to such a challenging task and a fundamental resource that allows for the successful and timely development of bioprocesses.

Process intensification to improve selectivity, yields, process control, safe operation modes, flexible production and cost effectiveness can be achieved by microreactor technology [7]. Both screening of production conditions (including selection of strains and medium composition) and downstream processes can be assessed within these reactors $[7,8]$. In this case, sub-optimal conditions of heat and mass transfer, in particular oxygen transfer, must be avoided by using proper engineering strategies [9]. The design of the bioreactors, modelling and prediction of operational conditions is thus of vital importance. The recent trend is to further miniaturize milliliter-scale reactors to the microliter scale, with the introduction of microstructured reactors [10]. Although all these platforms are clearly fit for high-throughput applications, limitations can be pointed out within them, such as: (i) limited culture volumes, (ii) medium evaporation and (iii) difficult process control. Furthermore, engineering characterization of these miniaturized devices is still far from being fully established [11].

Once appropriate operational modes are selected, a larger scale process is envisaged. This can be accomplished by two ways: (i) extrapolation by combination, e.g. assembling a large number of 
micro-reactors [10] or (ii) the process can be volumetrically scaledup using suitable criteria, e.g. constant oxygen transfer rate $\left(k_{L} a\right)$, maintenance of geometric parameters and constant volumetric power consumption (P/V) [12]. The former method enables high production volumes at relatively low cost as it is based on economyof-mass manufacturing while the latter is economy-of-scale based [10]. The study of the limiting steps will allow the full understanding of the economic viability of the process [8].

In the present case, a high-throughput strategy was used to develop a siderophore production process. Siderophores are highaffinity, low-molecular mass, iron-chelators, synthesized in nature by bacteria and fungi in very low quantities and, in industry, they are presently produced through chemical synthesis only [13]. In bacteria, the production of metal chelators is a survival strategy in environments presenting metals in ionization states (e.g. $\mathrm{Fe}^{3+}$ ) that are sparingly soluble and/or in a form that cannot be metabolized (e.g. iron hydroxides). High-affinity metal chelators can (i) decrease the concentration of metal ions when they are in excess [14], and (ii) increase the bioavailability of metal ions when they are scarce [15]. Examples of application of the former case include treatment of iron overload observed in transfusion dependent anaemic patients [14] and of accidental exposure to toxic metals [16]. Presently, there is an increased interest in metal chelators as therapeutic agents for diseases involving metal ion imbalance, such as, Friedreich's ataxia, and Wilson's and Alzheimer's diseases [17]. Furthermore, metal chelators have a wide field of applications in the industry and in metal remediation of contaminated sites [18].

In this work a combined high-throughput strategy was employed to screen a library of environmental bacteria for the assessment of high siderophore producers in order to shorten the gap among the early stages of process development and design. The results obtained in small-scale were scaled-up to lab scale stirred tank reactors (STR). To guarantee the industrial compatibility and viability of this bioprocess, three pre-established conditions were chosen: (i) overproduction of siderophore compared to the laboratory reference strain, (ii) production capabilities at $28-30^{\circ} \mathrm{C}$ and $\mathrm{pH}$ 6-8 and (iii) ultimately the bacterial strain must be non-pathogenic.

\section{Materials and methods}

\subsection{Materials}

Chrome azurol S (CAS), hexadecyltrimethylammonium bromide (HDTMA), 8hydroxyquinoline, 5-sulfosalicylic acid and piperazine were purchased from Sigma (St Louis, MO, USA). Tryptic Soy Agar (TSA) was purchased from Fluka (Deisenhofen, Germany). Iron free Milli-Q water (Millipore, MA, USA) was prepared using $1 \%(w / v)$ 8 -hydroxyquinoline in chloroform to perform a liquid-liquid extraction [19]. This iron free water was used to prepare all media and reagents. Glassware was acidwashed with 3 M HCI. The SensorDish ${ }^{\circledR}$ Reader, HydroDish ${ }^{\circledR}$ and OxoDish ${ }^{\circledR}$ MTP were from PreSens GmbH (Regensburg, Germany). HydroDish ${ }^{\circledR}$ plates presented a measurement range of $\mathrm{pH} 6-8.5$ with a resolution of +0.05 and accuracy of +0.1 at pH 7. OxoDish ${ }^{\circledR}$ had a measurement range of $0-250 \%$ air saturation with a resolution of $\pm 2 \%$ and accuracy of $\pm 5 \%$ at $100 \%$ air saturation. All other chemicals were of analytical or high-performance liquid chromatography (HPLC) grade, and purchased from various suppliers.

\subsection{Bacterial isolation}

Marine sediments samples were taken along the coast of Portugal during the months of May and June 2010 until a depth of $20 \mathrm{~m}$ with appropriate gear under sterile conditions. A volume of $100 \mu \mathrm{L}$, both from the liquid phase and sediments, was plated onto TSA plates (prepared according to manufacturer). Incubation was performed at $30^{\circ} \mathrm{C}$ for $24 \mathrm{~h}$. The grown microorganisms were further isolated in TSA plates and incubated at $30^{\circ} \mathrm{C}$ for $24 \mathrm{~h}$.

\subsection{Growth conditions}

\subsubsection{Inoculum preparation}

Bacterial growth was carried out in defined medium containing per liter of Milli-Q water the following: $15 \mathrm{~g}$ Casein peptone, $5 \mathrm{~g}$ Peptone, $24 \mathrm{~g} \mathrm{NaCl}, 6.5 \mathrm{~g}$ $\mathrm{MgSO}_{4} \cdot 7 \mathrm{H}_{2} \mathrm{O}, 5 \mathrm{~g} \mathrm{MgCl}_{2} \cdot 6 \mathrm{H}_{2} \mathrm{O}, 0.7 \mathrm{~g} \mathrm{KCl}$ and $0.1 \mathrm{~g} \mathrm{CaCl}_{2} \cdot 2 \mathrm{H}_{2} \mathrm{O}$ (final pH 7). The growth of inocula was performed at $30^{\circ} \mathrm{C}$ in $100 \mathrm{~mL}$ Erlenmeyer flasks, with a headspace of
$80 \%$, placed in orbital shakers (Agitorb, Aralab, Portugal) with $25 \mathrm{~mm}$ shaking diameter, at a shaking frequency of $200 \mathrm{rpm}$. A volume corresponding to $10 \%(\mathrm{v} / \mathrm{v})$ of the final volume, was transferred to the growth/production medium, once an optical density (OD) of roughly $0.7(640 \mathrm{~nm})$ was reached. Stock cultures were prepared by adding $250 \mu \mathrm{L}$ of the previous cultured bacteria to $250 \mu \mathrm{L}$ glycerol in 96 deep well microtiter plates which were stored at $-80^{\circ} \mathrm{C}$.

\subsubsection{Siderophore production}

Runs in microtiter plates were carried out in 24-well SensorDish ${ }^{\circledR}$ Reader HydroDish $^{\circledR}$ (for $\mathrm{pH}$ monitoring) and OxoDish ${ }^{\circledR}$ (for dissolved oxygen monitoring) from PreSens GmbH (Germany). Each well was filled with $0.5 \mathrm{~mL}$ of growth/bioconversion media, and sealed with tapes (Excel Scientific, CA, USA). The filling volume already includes the volume of inocula. Shaking frequency was set at $200 \mathrm{rpm}$ in Agitorb orbital shakers (Aralab, Portugal) with a $25 \mathrm{~mm}$ shaking diameter.

Bench-scale batch runs were carried out in a $5 \mathrm{~L}$ stirred tank reactor (Biostat B, B. Braun, Germany) with two Rushton turbines. Growth and/or production were performed in the defined medium listed previously under the following general conditions: medium volume $4 \mathrm{~L}$, aeration rate of $1 \mathrm{vvm}$, and stirring speed of $475 \mathrm{rpm}$. Temperature, initial $\mathrm{pH}$, medium composition and inoculation volume $(10 \% \mathrm{v} / \mathrm{v})$, were similar to the shaken systems.

Growth was followed off-line by OD $(640 \mathrm{~nm})$ and dry weight, applying a sacrificial well approach in the case of the MTP [20].

\subsubsection{Siderophore detection}

Siderophores were detected in CAS agar plates, prepared according to Schwyn and Neilands [21], by inoculating the stock bacteria with the aid of the Cryoreplicator press (EnzyScreen BV, The Netherlands). Additionally to the 95 bacteria strains inoculated, a reference strain (Mycobacterium smegmatis) was added to the plate. The plates were incubated at $30^{\circ} \mathrm{C}$ and monitored by image analysis every $24 \mathrm{~h}$.

In liquid cultures, the bacterial cells were recovered by centrifugation and the supernatant was analysed for the presence of siderophore [21]. Simultaneously, the SideroTec kit (Maynooth, Ireland; http://www.emergenbio.com/) was used, according to the manufacturers' instructions, as an alternative high-throughput detection and quantification method.

\subsection{High throughput image analysis}

At least 3 pictures of the CAS plates were taken with a Nikon Coolpix P5100 camera every $24 \mathrm{~h}$. The camera had a $7.5-26.3 \mathrm{~mm}(1: 2.7-5.3)$ zoom lens and was set at the macro function. Lens aperture was set to have as little depth-of-field as possible. All images were taken at the same magnification in the red-green-blue (RGB) system after white calibration to the local light conditions. Image analysis was carried out using the software Image-Pro Plus (Media Cybernetics, Inc., USA). The initial RGB image was segmented into the respective Red, Blue and Green channel images which were adjusted in terms of brightness and contrast. The Red and Blue channel images were used to assess siderophore and bacterial presence, respectively. Object size in images was measured after calibration and conversion of the pixel size to the metric system, using the measurements command.

\subsection{Bacterial identification}

Bacterial strains were identified using the Sherlock ${ }^{\circledR}$ Microbial ID System (MIS) from MIDI, Inc. (Newark, DE, USA). Cellular fatty acids, from cells grown on TSA plates at $30^{\circ} \mathrm{C}$, were extracted and methylated using the Instant $\mathrm{FAME}^{\mathrm{TM}}$ procedure. The fatty acid methyl esters (FAMEs) were analysed on a $6890 \mathrm{~N}$ gas chromatograph (GC) from Agilent Technologies (Palo Alto, CA, USA), with a FID and a 7683 B series injector, equipped with a $25 \mathrm{~m}$ long Agilent J\&W Ultra 2 capillary column from Agilent. The GC was programmed and controlled by the MIDI Sherlock software package, version 6.1. The FAMEs were identified by the MIDI software using MIDI calibration standards.

\subsection{Oxygen mass transfer coefficients}

Oxygen mass transfer coefficients $\left(k_{L} a\right)$ in the reactors used were obtained by using the gassing-out method. In the STR, deaeration was accomplished by flushing the reactor with nitrogen and in the MTP by applying the sulfite method according to John et al. [22].

In the MTP, $k_{L} a$ was determined for a shaking diameter of $25 \mathrm{~mm}$ at shaking frequencies ranging from 0 to $300 \mathrm{rpm}$ and $0.5 \mathrm{~mL}$ filling volume. In the STR, stirring frequencies ranged from 150 to $600 \mathrm{rpm}$ with the distances between the two Rushton turbines being $8.8 \mathrm{~cm}$ and a filling volume of $4 \mathrm{~L}$ with aeration rate of $1 \mathrm{vvm}$ was used.

\subsection{Statistical analysis}

Data were analysed using statistical analysis software (SPSS 14.0). The statistical treatment was obtained by one-way ANOVA, which was used to detect differences among variables. Statistical confidence was set at $95 \%$. 
A

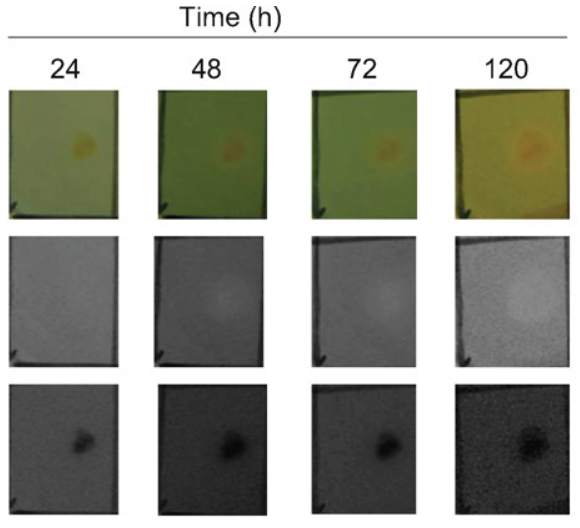

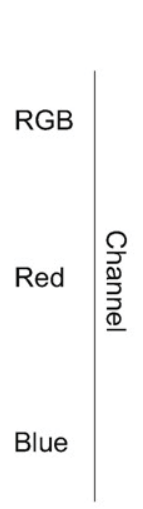

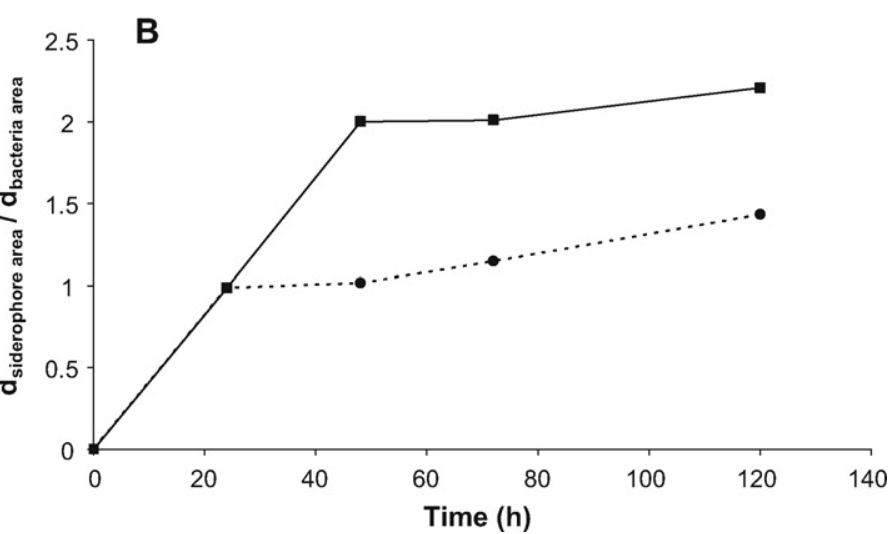

Fig. 1. (A) Image analysis of CAS plate in RGB, Red (siderophore) and Blue (bacteria) channels. (B) Correlation between the diameter of the area corresponding to siderophore production and the diameter of the colony of strain SD4 (solid line) and reference strain (dashed line). (For interpretation of the references to color in this figure legend, the reader is referred to the web version of the article.)

\section{Results and discussion}

\subsection{Screening for siderophore production capability}

In order to select the desired siderophore producers, the samples collected along the Portuguese Coast were initially plated into TSA medium. This medium assured the growth of all environmental bacteria at the pre-established conditions $\left(30^{\circ} \mathrm{C}, \mathrm{pH}\right.$ 7.0). Samples from both the sediments and the aqueous phase were plated for microbial isolation, and the ratio of the number of colonies observed in the sediments: aqueous phase was roughly 5:1 (not taking into account fungi and algae growth). In total, c.a. 500 microorganisms were isolated.

The first selection step assured the elimination of fungus and microalgae. The colonies corresponding to bacteria were spread in TSA plates for isolation and were analysed according to the Sherlock ${ }^{\circledR}$ Microbial ID System from MIDI, Inc. [23,24]. After identification, bacteria with risk factor higher than 1 were discarded. At the end of this procedure, 136 bacterial candidates were obtained. Strains belonging to the same species according to the MIDI system were maintained in the candidate list since intrinsic metabolic characteristics can be responsible for different capabilities.

The bacterial candidates were plated onto CAS agar plates with the aid of a cryo-replicator press. This assured a uniform spatial distribution of the samples as well as standardized plating, so critical for image analysis. It is of paramount importance to incorporate into the CAS plate a reference strain. This will insure the uniformity of all data collected from this platform. In the present work, the reference strain used was Mycobacterium smegmatis an exochelin MS producer [25] regularly used in our laboratories.

The plates were photographed every day and evaluated by image analysis. Similar strategies for high throughput determination had been performed previously [26] where biomass growth was assessed in microtiter plates by correlating the area occupied by biomass with the optical density (OD) of the cell suspension. Combining this with the conclusions taken by Shin and co-workers, who evidenced that there is a linear relationship between the concentration of siderophores and the diameter of haloes obtained by diffusion, the throughput of the CAS agar test was increased significantly [27]. Nonetheless, despite the large number of assays, the traditional CAS agar test gives only qualitative results in terms of siderophore production.

The initial pictures were taken in the RGB colour system (Fig. 1A). By isolating the images of the Red and Blue channels, the visualization of, respectively, the siderophore haloes and the bacterial colonies was significantly improved. By computing a
Table 1

Siderophores producers identified by the Sherlock ${ }^{\circledR}$ Microbial ID System from MIDI, Inc.

\begin{tabular}{lll}
\hline Code & Identified strain & Sampling location \\
\hline AA1, AA2 & Pseudomonas putida & Aveiro lagoon \\
AB1, AH3 & Pseudomonas fluorescence & Aveiro lagoon \\
AD3 & Serratia odorifera & Aveiro lagoon \\
CA3 & Bacillus megaterium & Carcavelos beach shore \\
SA2 & Staphylococcus sciuri & Tagus River delta - Seixal \\
SD1 & Micrococcus lylae & Tagus River delta - Seixal \\
SD2, SD3 & Brevibacillus laterosporus & Tagus River delta - Seixal \\
SD4 & Kocuria rhizophila & Tagus River delta - Seixal \\
\hline
\end{tabular}

relationship between both diameters is was possible to compare our reference strain with the library strains in terms of siderophore production (Fig. 1B). A library strain was considered to have overproduction capabilities if the correlation between diameters was higher than $10 \%$ compared to the reference strain. The combined use of the image analysis and the high throughput CAS platform allowed the reduction of the 136 initial candidate strains to only 11 strains which were able to produce higher amounts of siderophores than the reference strain. The siderophore producers are listed in Table 1.

For some of these strains, this work is the first evidence that they are siderophore producers. In fact, and to our knowledge, Serratia odorifera and Micrococcus lylae had not been previously identified as able to produce siderophores. Further studies are envisaged to fully characterize siderophore production in these strains.

Although the traditional CAS assay is a reliable and established method, a new high throughput quantification method is necessary for reducing both time and sample volume required and also to improve the sensitivity of analysis. This is of vital importance in a screening process where, in most cases, the volumes employed are often in the range of the $\mu \mathrm{L}$ [7]. The SideroTec assay tested in the present study allowed to obtain quantification data in roughly 15 min (with no previous preparation of samples) while the standard CAS assay [21] can provide results after at least $3 \mathrm{~h} \mathrm{[28]} \mathrm{with}$ volume of samples higher than $50 \mu \mathrm{L}$.

When the SideroTec kit was compared to the standard liquid CAS assay, two distinct regions were observed in the plot (Fig. 2). For low concentrations of siderophores $\left(0-0.15 \mathrm{mg} \mathrm{mL}^{-1}\right)$ the kit gives more reliable results than the traditional CAS method, since the traditional CAS method can not distinguish siderophore concentrations in the range of $0.1 \mathrm{ml} \mathrm{mL}^{-1}$. This could be observed by the divergent curve behaviour of the calibrations (data not 
A

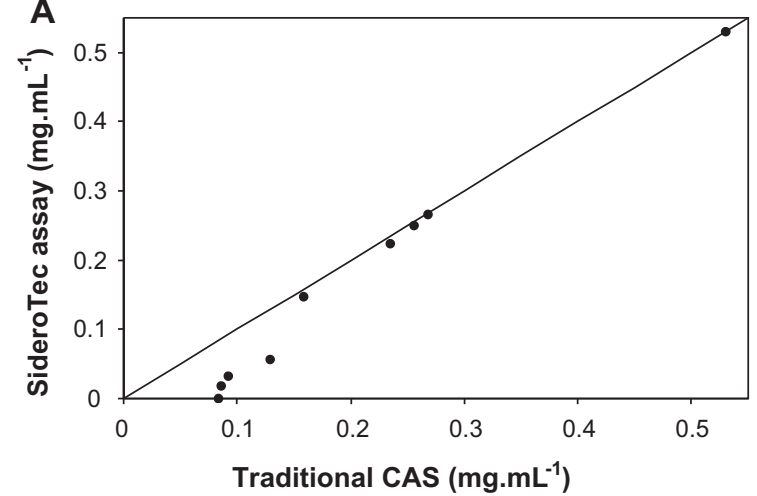

B

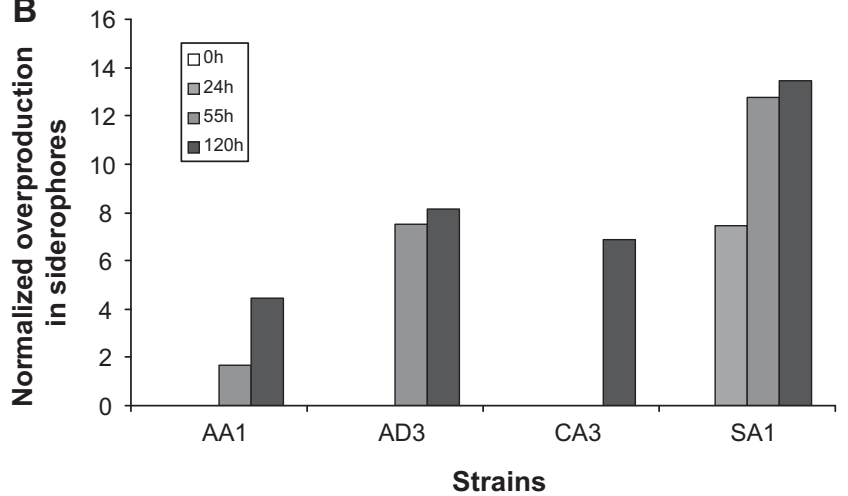

Fig. 2. (A) Correlation between the SideroTec assay and the traditional CAS quantification method. (B) Quantification of siderophore production of different bacteria strains using the SideroTec kit.

shown). Concerning the region of concentrations between 0.15 and $1.0 \mathrm{mg} \mathrm{mL}^{-1}$ the two methods give similar results (Fig. 2).

\subsubsection{Assessing growth}

The growth and production conditions of the isolated strains selected by the high throughput CAS agar method were evaluated by using the SensorDish ${ }^{\circledR}$ Reader system. This system comprises microtiter plates with incorporated sensors in the wells for $\mathrm{pH}$ and dissolved oxygen measurements. Nonetheless, quantification of most analytes requires off-line analysis. Accordingly, in this particular study and set-up, siderophore production was assessed off-line by the SideroTec assay. Particular attention was given to the volumes of the samples due to the already small volume of the growth media used. Sealing tapes were used to prevent evaporation of media and a sacrificial well approach was employed.

Regarding the dissolved oxygen concentration during growth, there are clearly three different regions along the bacterial growth curves, corresponding to the lag, exponential growth and stationary phases (Fig. 3A). These phases can vary in duration depending on the selected strain and growth conditions. A longer lag phase indicates possibly longer adaptation towards medium stress conditions (e.g. lack of iron). The exponential growth phase can be easily identified in the microtiter plate system by the corresponding fast oxygen depletion observed, its duration depending on the oxygen demand of the individual strains. Afterwards, the stationary phase can be observed by an increase in dissolved oxygen (DO).

Similarly, three different growth phases could be observed in the biomass profile (Fig. 3B). Previously, other authors have shown correspondence between DO profiles and biomass production [29-33], suggesting that bacterial growth may be followed without the need for sampling or for interfering with the system. However, there were some discrepancies in the observed trends in biomass and DO in the case of M. smegmatis (Fig. 3) although a mandatory calibration between $\mathrm{pO}_{2}$ measurements and biomass production (measured by OD or dry weight) was previously performed. Phenomena like medium evaporation or low oxygen uptake rates, dependent on bacterial metabolism, can be in the origin of these discrepancies.

In the end of process development, if the siderophore production data and the data collected with the monitored microtiter plates are overlapped, the bioprocess itself can be tuned to, e.g. type of siderophore, production titer or overall production time.

\subsection{Scale-up of bioproduction}

Bioproduction runs were performed in $\mathrm{pH}$ - and oxygenmonitored MTP and in a 5-L stirred reactor maintaining the same $k_{L} a$ value in both systems (Fig. 4). This criterion is regularly used for scaling-up bioprocess as shown in recent reviews and references therein $[11,34,35]$ and was chosen since the bioprocess tested was based on aerobic growing bacterial cells in a simple aqueous system. The $k_{L} a$ was set at $0.044 \mathrm{~s}^{-1}$ which corresponds to a shaking frequency of $200 \mathrm{rpm}$ in the MTP system and to $475 \mathrm{rpm}$ in the STR. No significant alteration in $k_{L} a$ profiles $(<10 \%)$ were observed when compared to similar studies carried out previously $[29,30]$. These stirring speeds were maintained along the bioproduction runs. Moreover, since (i) the runs were performed using a low viscosity medium, (ii) no high bacterial density values were reached and (iii) no out-of-phase conditions were observed [36], it was considered that $k_{L} a$ was maintained at $0.044 \mathrm{~s}^{-1}$ in both reactors along the runs.
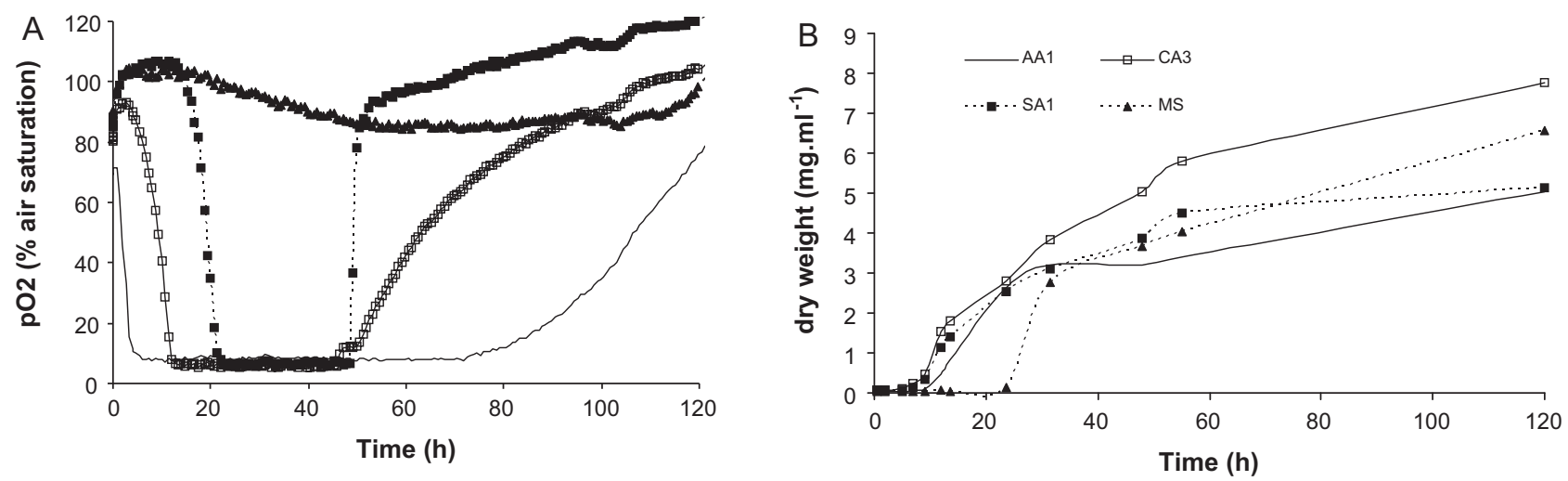

Fig. 3. (A) Oxygen profile measured within the SensorDish ${ }^{\circledR}$ Reader OxoDish ${ }^{\circledR}$ for different strains at $30{ }^{\circ} \mathrm{C}$ and $200 \mathrm{rpm}$. (B) Biomass profile measured off-line for different bacterial strains. 

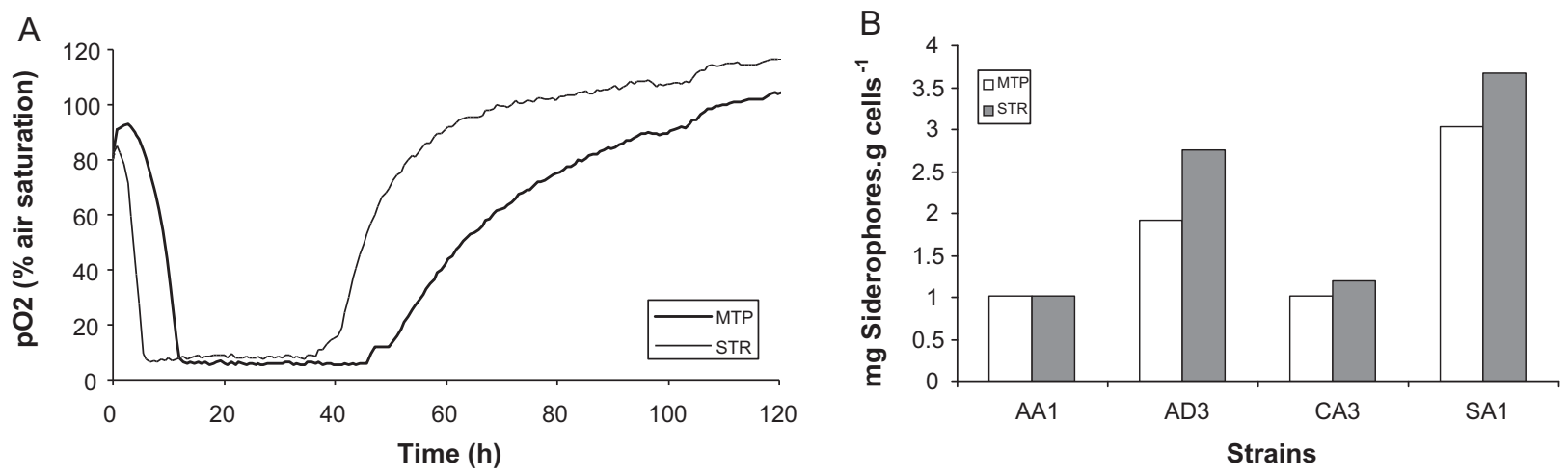

Fig. 4. (A) Oxygen consumption profiles of strain AD3 in microtiter plate (MTP) and in a $5 \mathrm{~L}$ stirred tank reactor (STR). (B) Siderophore production titration quantified by the SideroTec assay for the different strains in MTP and STR at $120 \mathrm{~h}$.

On-line data for oxygen consumption were collected for MTP and compared to data gathered with the 5-L bench-scale reactor. Comparative data from the two scales are given for strain AD3 (Serratia odorifera) since it is the first reference, as far as the authors' knowledge, of siderophore production and bioprocess monitoring for this strain. The scale-up of the bioproduction allowed the production of the siderophore at the mg scale thus providing enough quantity for further chemical characterization studies. Concerning DO, there was an initial decay of oxygen in the $5 \mathrm{~L}$ stirred tank reactor, which was not observed in the MTP system (Fig. 4A). Still, the two DO profiles are not fully superimposable, a feature already observed previously when miniaturized reactors are compared to larger reactors [30,33,37]. Despite the evidences, justifications in the literature for these discrepancies are scarce. Previously, Micheletti et al. [38] showed the same divergence in cell growth curves when shaken systems (MTP and shaken flasks) were compared to STR in fermentation runs for the production E. coli JM107:pQR706 cells overexpressing transketolase. In shaken systems, a lag phase was also observed in the growth curves which was not visible in the STR.

Possibly heterogeneous environments (e.g. DO gradients) are involved since bacteria cells are continuously exposed to fluctuating conditions, that consequentially, subjected them to local environmental stresses causing different metabolic responses [39-41].

Siderophore titration (Fig. 4B) shows that scale-up strategy followed allowed identical production between the MTP and the $5 \mathrm{~L}$ STR. The proposed bioprocess development strategy allowed an efficient and rapid manner to screen for siderophore producers from a library of environmental bacteria. This strategy was based on the production of undifferentiated siderophore types. If a specific siderophore is desired, an extra step of characterization must be performed.

Further studies are envisaged to incorporate a high-throughput purification and characterization step into this Process Intensification Platform. This would increase the application of the approach, making it suitable to other molecules of therapeutic interest.

\section{Conclusions}

Eleven bacterial strains able to produce siderophores in significant quantities were identified from an initial library of 500 bacteria. The proposed high-throughput CAS agar plate analysis allowed the screening of siderophores from 96 bacterial strains simultaneously. Moreover, the use of microtiter plates with on-line measurement of oxygen allowed the rapid evaluation of production conditions, mimicking runs performed at bench-scale. Similar results were observed in MTP and STR in terms of specific product yields, while reducing costs related to equipment, reagents and handling in the MTP. Further improvements should include fully automated platforms with monitoring and control devices.

\section{Acknowledgements}

The authors acknowledge Fundação para a Ciência e a Tecnologia (FCT, Portugal) for financial support to C.C.C.R. de Carvalho and P. Fernandes under program Ciência 2007 and for the postdoctoral grant (SFRH/BPD/64160/2009) awarded to M.P.C. Marques and C.M. do Seixal for logistic support during sampling. K. Walshe and S. Doyle were funded by Enterprise Ireland (Grant No. EI/CFTD/2008/0141).

\section{References}

[1] Fernandes P, Cruz A, Angelova B, Pinheiro HM, Cabral JMS. Microbial conversion of steroid compounds: recent developments. Enzyme Microb Technol 2003;32:688-705.

[2] Fukuda H, Kondo A, Tamalampudi S. Bioenergy: sustainable fuels from biomass by yeast and fungal whole-cell biocatalysts. Biochem Eng J 2009;44:2-12.

[3] de Carvalho CCCR, da Fonseca MMR. Influence of reactor configuration on the production of carvone from carveol by whole cells of Rhodococcus erythropolis DCL14. J Mol Catal B: Enzym 2002;1:9-20, 377-87.

[4] Watts KT, Mijts BN, Schmidt-Dannert C. Current and emerging approaches for natural product biosynthesis in microbial cells. Adv Synth Catal 2005;347:927-40.

[5] de Carvalho CCCR. Enzymatic and whole cell catalysis: finding new strategies for old processes. Biotechnol Adv 2011;29:75-83.

[6] de Carvalho CCCR, Poretti A, da Fonseca MMR. Cell adaptation to solvent, substrate and product: a successful strategy to overcome product inhibition in a bioconversion system. Appl Microbiol Biotechnol 2005;69:268-75.

[7] Marques MP, Cabral JMS, Fernandes P. High throughput in biotechnology: from shake-flasks to fully instrumented microfermentors. Recent Patent Biotechnol 2009;3:120-40.

[8] Titchener-Hooker NJ, Dunnill P, Hoare M. Micro biochemical engineering to accelerate the design of industrial-scale downstream processes for biopharmaceutical proteins. Biotechnol Bioeng 2008;100:473-87.

[9] Büchs J. Introduction to advantages and problems of shaken cultures. Biochem Eng J 2001;7:91-8.

[10] Saber M, Commenge JM, Falk L. Microreactor numbering-up in multi-scale networks for industrial-scale applications: impact of flow maldistribution on the reactor performances. Chem Eng Sci 2010;65:372-9.

11] Marques MPC, Cabral JMS, Fernandes P. Bioprocess scale-up: quest for the parameters to be used as criterion to move from microreactors to lab-scale. J Chem Technol Biotechnol 2010;85:1184-98.

[12] Schmidt F. Optimization and scale up of industrial fermentation processes. Appl Microbiol Biotechnol 2005;68:425-35.

13] Geraci LS, Levy SG, Hudspeth JP, Buswell RL, Stearns JF. Chemical synthesis of exochelins. US Patent 2002, 6,335,443.

[14] Vermylen C. What is new in iron overload. Eur J Pediatr 2008;167:377-81.

[15] Neilands JB. Siderophores: function of microbial iron transport compounds. J Biol Chem 1995;270:26723-6.

[16] Andersen O. Chemical and biological considerations in the treatment of metal intoxications by chelating agents. Mini Rev Med Chem 2004;4:11-21.

[17] Bolognin S, Drago D, Messori L, Zatta P. Chelation therapy for neurodegenerative diseases. Med Res Rev 2009;29:547-70. 
[18] Leštan D, Luo C-l, Li X-d. The use of chelating agents in the remediation of metal-contaminated soils: a review. Environ Pollut 2008;153:3-13.

[19] Moeller T. Extraction and colorimetric estimation of certain metals as derivatives of 8-hydroxyquinoline. Ind Eng Chem Anal Ed 1943:15:346-9.

[20] Doig SD, Pickering SCR, Lye GJ, Woodley JM. The use of microscale processing technologies for quantification of biocatalytic Baeyer-Villiger oxidation kinetics. Biotechnol Bioeng 2002;80:42-9.

[21] Schwyn B, Neilands JB. Universal chemical assay for the detection and determination of siderophores. Anal Biochem 1987;160:47-56.

[22] John GT, Klimant I, Wittmann C, Heinzle E. Integrated optical sensing of dissolved oxygen in microtiter plates: a novel tool for microbial cultivation. Biotechnol Bioeng 2003;81:829-36.

[23] Buyer JS. Identification of bacteria from single colonies by fatty acid analysis. J Microbiol Methods 2002:48:259-65.

[24] Buyer JS. Rapid sample processing and fast gas chromatography for identification of bacteria by fatty acid analysis. J Microbiol Methods 2002;51:209-15.

[25] Sharman GJ, Williams DH, Ewing DF, Ratledge C. Isolation, purification and structure of exochelin MS, the extracellular siderophore from Mycobacterium smegmatis, Biochem J 1995:1:187-96.

[26] de Carvalho CCCR, Marques MPC, Fernandes P, da Fonseca MMR. A simple imaging method for biomass determination. J Microbiol Methods 2005;60:135-40.

[27] Shin SH, Lim Y, Lee SE, Yang NW, Rhee JH. CAS agar diffusion assay for the measurement of siderophores in biological fluids. J Microbiol Methods 2001:44:89-95.

[28] Park JH, Bolan N, Megharaj M, Naidu R. Isolation of phosphate solubilizing bacteria and their potential for lead immobilization in soil. J Hazard Mater 2011;185:829-36.

[29] Marques MPC, Cabral JMS, Fernandes P. A microwell platform for the scaleup of a multistep bioconversion to bench-scale reactors: sitosterol side-chain cleavage. Biotechnol J 2010;5:402-12.

[30] Marques MPC, Magalhães S, Cabral JMS, Fernandes P. Characterization of 24well microtiter plate reactors for a complex multistep bioconversion: from sitosterol to androstenedione. J Biotechnol 2009;141:174-80.

[31] Kensy F, Engelbrecht C, Buchs J. Scale-up from microtiter plate to laboratory fermenter: evaluation by online monitoring techniques of growth and protein expression in Escherichia coli and Hansenula polymorpha fermentations. Microb Cell Fact 2009;8:68

[32] Funke M, Buchenauer A, Mokwa W, Kluge S, Hein L, Muller C, et al. Bioprocess control in microscale: scalable fermentations in disposable and user-friendly microfluidic systems. Microb Cell Fact 2010;9:86.

[33] Islam RS, Tisi D, Levy MS, Lye GJ. Scale-up of Escherichia coli growth and recombinant protein expression conditions from microwell to laboratory and pilot scale based on matched $k_{L} a$. Biotechnol Bioeng 2008;99:1128-39.

[34] Garcia-Ochoa F, Gomez E. Bioreactor scale-up and oxygen transfer rate in microbial processes: an overview. Biotechnol Adv 2009;27:153-76.

[35] Garcia-Ochoa F, Gomez E, Santos VE, Merchuk JC. Oxygen uptake rate in microbial processes: an overview. Biochem Eng J 2010;49:289-307.

[36] Büchs J, Lotter S, Milbradt C. Out-of-phase operating conditions, a hitherto unknown phenomenon in shaking bioreactors. Biochem Eng J 2001;7:135-41.

[37] Gill NK, Appleton M, Baganz F, Lye GJ. Quantification of power consumption and oxygen transfer characteristics of a stirred miniature bioreactor for predictive fermentation scale-up. Biotechnol Bioeng 2008;100:1144-55.

[38] Micheletti M, Barrett T, Doig SD, Baganz F, Levy MS, Woodley JM, et al. Fluid mixing in shaken bioreactors: implications for scale-up predictions from microlitre-scale microbial and mammalian cell cultures. Chem Eng Sci 2006;61:2939-49.

[39] Lara AR, Leal L, Flores N, Gosset G, Bolivar F, Ramirez OT. Transcriptional and metabolic response of recombinant Escherichia coli to spatial dissolved oxygen tension gradients simulated in a scale-down system. Biotechnol Bioeng 2006;93:372-85.

[40] Lara AR, Taymaz-Nikerel H, Mashego MR, van Gulik WM, Heijnen JJ, Ramirez OT, et al. Fast dynamic response of the fermentative metabolism of Escherichia coli to aerobic and anaerobic glucose pulses. Biotechnol Bioeng 2009;104: 1153-61.

[41] Lara AR, Galindo E, Ramirez OT, Palomares LA. Living with heterogeneities in bioreactors: understanding the effects of environmental gradients on cells. Mol Biotechnol 2006:34:355-81. 